\title{
Acompanhamento do crescimento dental em Puma concolor mantido em cativeiro ${ }^{1}$
}

\author{
João L. Rossi Junior ${ }^{2}$, Marco A. Gioso ${ }^{2}$, Marcelo Q. Teles ${ }^{3}$ e Léslie M. \\ Domingues-Falqueiro ${ }^{4}$
}

\begin{abstract}
Rossi Jr J.L., Gioso M.A., Teles M.Q. \& Domingues-F. L.M. 2007. [Accompaniment of the dental growth in Puma concolor kept in captivity.] Acompanhamento do crescimento dental em Puma concolor mantido em cativeiro. Pesquisa Veterinária Brasileira 27(5):205-208. Departamento de Cirurgia da Faculdade de Medicina Veterinária e Zootecnia, Universidade de São Paulo, Av. Prof. Dr. Orlando de Marques de Paiva 87, Bloco 8-superior, Cidade Universitária, São Paulo, SP 05508-270, Brazil. E-mail: vetjrossi@gmail.com

The development of dentistry is delayed in the preventive internal medicine for wild animals. Some international papers about wild animal dentistry have already been published regarding some species in wild life or kept in captivity in different geographical regions, but not specifically about the great neotropical felines Panthera onca and Puma concolor. The aim of this study was to survey the prevalence of malocclusion in neotropical felines maintained in the state of São Paulo. For the study a sample of 42 jaguars (Panthera onca) and 36 pumas (Puma concolor) was used, totalizing 78 animals, proceeding from 18 institutions where such species were kept in captivity. All animals were examined if the occlusion was in accordance with the normal for the species. The teeth were identified one by one, examined directly by means of a dental explorer. Malocclusion was present in 47.61\% (20 cases) in Panthera onca, and in 5.55\% ( 2 cases) of Puma concolor. The animals with malocclusions apparently did not have problems of food apprehension or chewing ability, although they presented a more reduced interproximal space, with food accumulation the lack of removal of which could provoke oral problems.
\end{abstract}

INDEX TERMS: Felidae, Puma concolor, captivity animals, stomatognathic system.

RESUMO.- Objetivou-se neste trabalho fazer levantamento sobre a troca de dentição decídua por permanente, notadamente dos dentes caninos e a prevalência de maloclusão em pumas (Puma concolor) manejados nas instituições visitadas no Estado de São Paulo. Para os estudos utilizou-se amostra constituída de 36 pumas, provenientes de 18 instituições mantenedoras de tais espécies em cativeiro no Estado de São Paulo, sendo que três animais com idade de oito meses, irmãos de ninhada, apresentaram retenção dos dentes caninos decíduos e foram acompanha-

\footnotetext{
${ }^{1}$ Recebido em 7 de fevereiro de 2007.

Aceito para publicação em 11 de maio de 2007.

${ }^{2}$ Departamento de Cirurgia, Faculdade de Medicina Veterinária e Zootecnia (FMVZ), Universidade de São Paulo (USP), Av. Prof. Dr. Orlando de Marques de Paiva 87, Bloco 8-superior, Cidade Universitária, São Paulo, SP 05508-000, Brasil. *Autor para correspondência: vetjrossi@gmail.com

${ }^{3}$ Médico Veterinário do Zoológico Municipal Altino Müller, Av. Prefeito José Losano de Araújo 1515, Jd. N.S.Aparecida, Paulínia, SP 13140-000.

${ }^{4}$ Pós-Doutoranda do Departamento de Cirurgia, FMVZ, USP.
}

dos por 2 anos e 8 meses. Todos os animais foram examinados, observando se a oclusão estava de acordo com o normal para a espécie. Os dentes foram identificados um a um, examinados diretamente por meio de explorador odontológico. Os animais que apresentaram retenção dos dentes caninos decíduos não foram tratados, pois a maloclusões aparentemente não comprometia a preensão ou mastigação de alimentos, embora apresentassem acúmulo de alimentos ou indutos moles na região dos dentes com espaço interproximal mais reduzido.

TERMOS DE INDEXAÇÃO: Felidae, Puma concolor, animais de cativeiro, sistema estomatognático.

\section{INTRODUÇÃO}

As doenças da cavidade oral em animais selvagens não costumam ser bem compreendidas e documentadas por profissionais que trabalham com tais espécies (Kazimiroff 1938, Robinson, 1979). As infecções da cavidade oral podem estar diretamente relacionadas com enfermidades dentais primárias como: erup- 
ção dental deficiente, maloclusão, desgaste e abrasão precoce, fraturas com ou sem exposição de pulpar e doença periodontal.

Puma concolor tem ampla distribuição latitudinal, abrangendo assim, os mais diversos habitats ocorrendo desde o Canadá até o Chile, incluindo também o Brasil (Redford \& Eisenberg 1992).

A União Internacional para Conservação da Natureza e dos Recursos Naturais (IUCN) lista as subespécies Puma concolor cougar e Puma concolor coryi como criticamente ameaçadas. No Chile o puma é tido como "vulnerável" na maioria das regiões, mas ao norte é considerado "ameaçada". A Lista dos Animais Ameaçados de Extinção (IBAMA) não faz distinção entre as diferentes subespécies, classificando todas como de menor preocupação (Leite \& Paula 2002).

Wiggs \& Lobprise (1997) relatam que as onças-pardas possuem aproximadamente os seguintes tempos de erupção dos dentes decíduos (Quadro 1).

Apesar destes autores referenciarem as idades possíveis para erupção dos dentes decíduos, não há referências sobre a idade com que estes dentes esfoliam e dão lugar aos dentes permanentes, tais pesquisas foram melhores conduzidas em primatas criados para uso em pesquisas Amand \& Tinkelman (1985). Gioso (2007) considera que o tempo médio para a troca da dentição decídua pela permanente nos felídeos silvestres é relativamente próximo ao dos felinos domésticos (Felis catus). Wiggs \& Lobprise (1997) reportaram o tempo de erupção dos dentes permanentes de uma outra espécie de felídeo, os linces (Lynx sp.) (Quadro 2).

Quadro 1. Tempo médio de erupção dos dentes decíduos (idade em dias) em Puma concolor

\begin{tabular}{cc}
\hline Dente & Dias de idade \\
\hline $1^{\circ}$ incisivo & $14-17$ \\
$2^{\circ}$ incisivo & $17-20$ \\
$3^{\circ}$ incisivo & $23-28$ \\
Canino & $28-29$ \\
$2^{\circ}$ pré-molar & - \\
$3^{\circ}$ pré-molar & $40-42$ \\
$4^{\circ}$ pré-molar & $37-47 /$
\end{tabular}

Fonte: Wiggs \& Lobprise (1997).

Quadro 2. Tempo médio erupção dos dentes permanentes (idade em dias) em Lynx sp.

\begin{tabular}{lc}
\hline Dente & Dias de idade \\
\hline Incisivos & $130-160$ \\
Caninos & 190 \\
Erupção mandibular completa & 240 \\
Erupção maxilar completa & 210
\end{tabular}

Fonte: Wiggs \& Lobpreise (1997), modificado.

A maloclusão pode não causar ou resultar necessariamente em nenhum problema, portanto, o tratamento nem sempre é necessário (Wiggs \& Lobprise 1997). Tentativas de tratamento ortodôntico em espécies selvagens podem ser complicadas, além do que ajustes e manutenção são usualmente difíceis (Wiggs \& Lobprise 1997).

É interessante notar que as causas de maloclusão nas espécies domésticas, mormente em cães, parece ser decorrência dos acasalamentos consangǘneos (Wiggs \& Lobprise 1997), com fins de modificar as dimensões corpóreas dos animais. Estes acasala- mentos acabam por diminuir o tamanho do crânio, mas não o dos dentes, proporcionalmente, levando a modificações na forma do arco dental dos animais. Problemas de oclusão não são relatados em animais selvagens, além dos aqui descritos, embora o sejam em carnívoros domésticos (Wiggs \& Lobprise 1997).

Objetivou-se neste trabalho fazer o acompanhamento odontológico de pumas mantidos em cativeiro, sendo que em três indivíduos da espécie Puma concolor, nascidos em cativeiro no Estado de São Paulo, observou-se o crescimento dental.

\section{MATERIAL E MÉTODOS}

Para os estudos utilizou-se amostra constituída 36 pumas (Puma concolor), provenientes de 18 instituições mantenedoras em cativeiro no Estado de São Paulo, sendo que três animais tinham 8 meses de idade, e foram acompanhados durante 2 anos e 7 meses.

Os animais mantidos em cativeiro foram avaliados clinicamente após a realização de contenção química do tipo dissociativa (Telazol ${ }^{\circledR}$, Fort Dodge, $6,0 \mathrm{mg} / \mathrm{kg}$, via intramuscular, através de disparo de dardo por zarabatana). Os três pumas irmãos de ninhada foram avaliados aos 8 meses de idade e posteriormente dois deles aos 2 anos e 8 meses (um dos filhotes morreu aos 1 ano e 3 meses de idade).

Foram realizados os levantamentos preliminares das condições da integridade da cavidade oral através de preenchimento de odontogramas e realização de documentação fotográfica e de filmagem da cavidade oral dos animais manejados.

Os animais foram examinados, observando se a oclusão estava de acordo com o normal para as espécies (Fig.1). Os dentes foram identificados um a um, examinados diretamente por meio de explorador odontológico. Para auxiliar aos exames procederam-se à documentação fotográfica e moldagem da cavidade oral por meio de silicone de adição $(3 \mathrm{M} \circledast)$. Os procedimentos realizados nos animais sempre contaram com a anuência e participação dos profissionais das instituições visitadas.

\section{RESULTADOS}

A permanência dos dentes caninos decíduos (Fig.2 e 3a,b) esteve presente em três filhotes de Puma concolor, com 8 meses de idade, comprovadamente irmãos de ninhada.

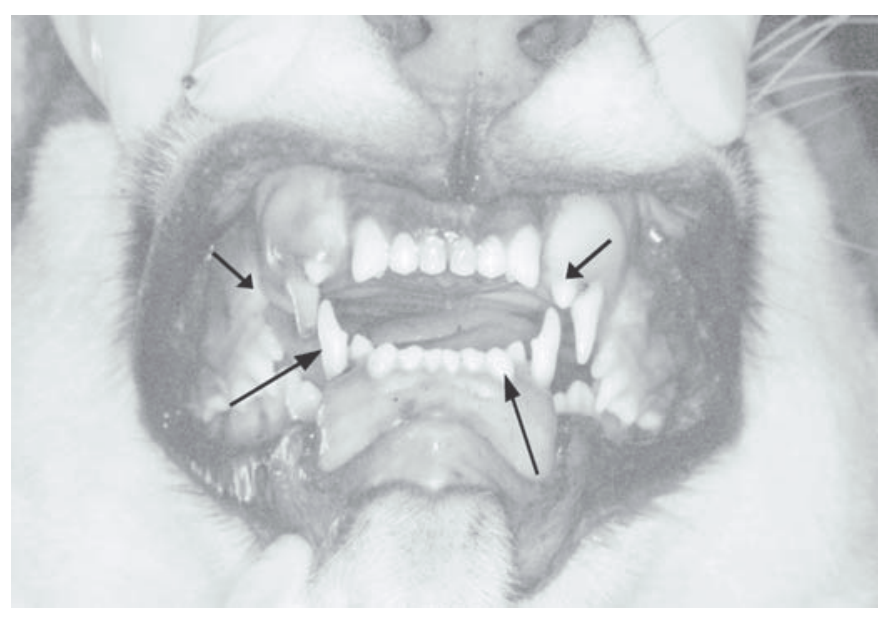

Fig.1. Aspecto da oclusão alterada de um filhote de Puma concolor com 8 meses de idade e retenção dos dentes decíduos. Os dentes caninos superiores estão extruindo do osso alveolar (setas curtas) e os dentes caninos inferiores permanentes apresentam deslocamento no sentido caudal aos dentes incisivos inferiores (setas longas), fazendo com que a sua oclusão seja palatina. 

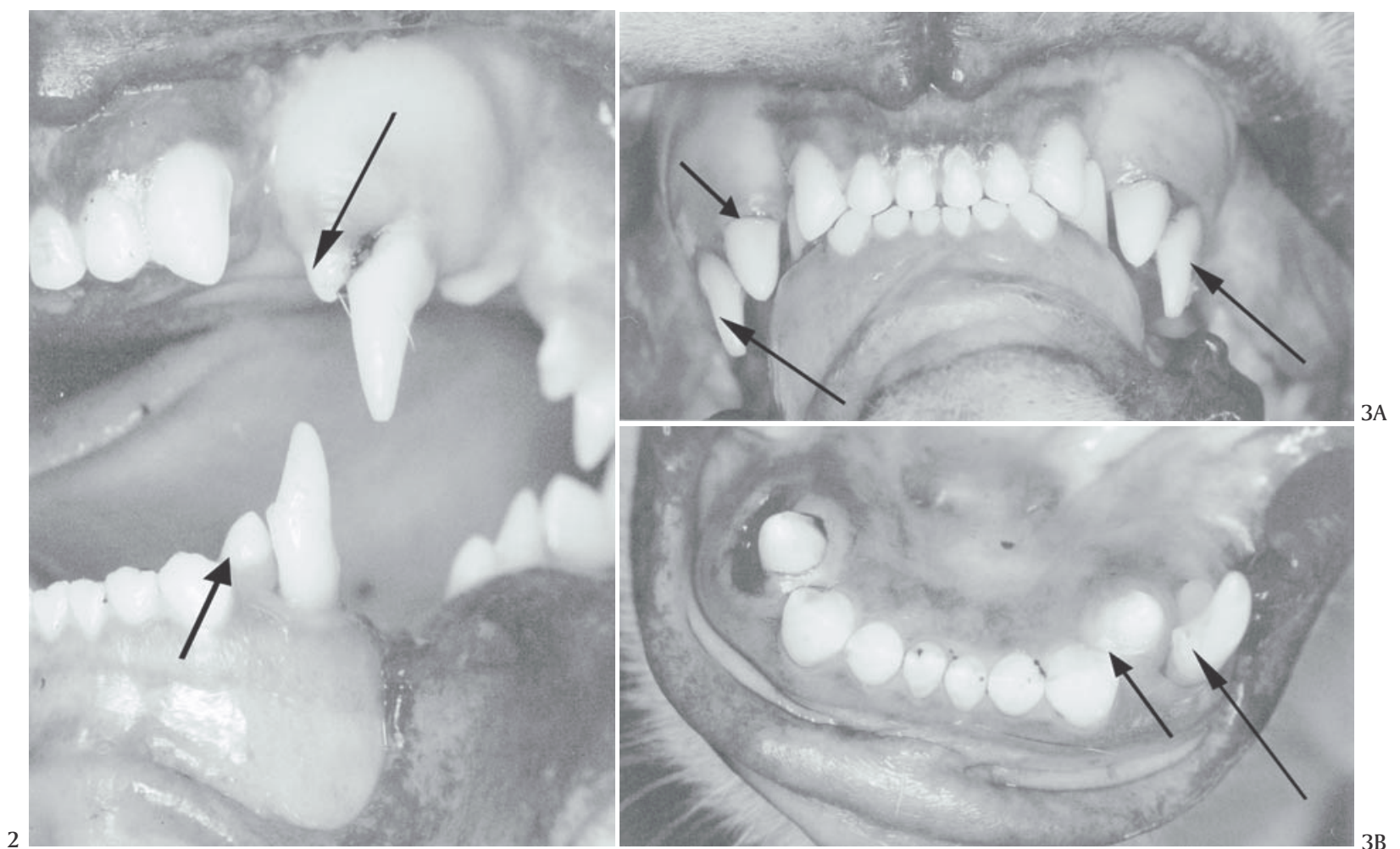

Fig.2. Posição caudal do dente canino inferior esquerdo e retenção do dente decíduo neste mesmo hemi-arco dental (seta curta). Pode-se notar a extrusão do dente canino superior no hemi-arco maxilar (seta longa).

Fig.3A. O dente canino inferior direito está ocluindo totalmente em posição aboral aos dentes incisivo lateral e canino superiores (seta curta). Nos dois hemi-arcos superiores nota-se a permanência dos dentes caninos decíduos (setas longas).

Fig.3B. Presença do dente canino inferior esquerdo decíduo e posicionamento do dente permanente no sentido lingual em relação ao dente incisivo inferior esquerdo (seta curta), e lingual ao canino decíduo (seta longa).

Observou-se em um filhote de $P$. concolor que o dente canino superior esquerdo decíduo apresentou fratura de cúspide com exposição de canal pulpar e escurecimento dental (Fig.4), antes do início da extrusão dos dentes caninos permanentes superiores.

Devido a não esfoliação dos dentes caninos, os achados de maloclusão relacionaram-se principalmente com o posiciona-mento vestibularizados dos dentes caninos permanentes superiores e o deslocamento dos dentes caninos permanentes inferiores para a posição ligual, fazendo com que a sua oclusão fosse palatina.

Em um dos animais avaliados observou-se a posição caudal do dente canino inferior esquerdo permanente e retenção do dente decíduo neste mesmo hemi-arco dental mandibular, fazendo com que o dente canino inferior direito ficasse em posição palatina aos dentes incisivo lateral e canino superiores.

Um dos animais pesquisados morreu com 1 ano e 3 meses de idade, e não apresentava maloclusão dos dentes caninos permanentes, verificada durante exame necroscópico.

Os dois outros animais foram avaliados com a idade de 2 anos e 8 meses e não apresentaram sinais de maloclusão dos dentes caninos (Fig.5-7). Não se tem resultados durante o intervalo de tempo entre o primeiro exame (aos 8 meses de idade) e aos 2 anos e 8 meses, pois não houve necessidade de realizar-se intervenções clínicas nos animais sob efeito de anestesia dissociativa.

\section{DISCUSSÃO}

Tais achados para Puma concolor não estão descritos em literatura, embora existam relatos de que $5-12 \%$ dos mamíferos selvagens apresentam maloclusão, podendo chegar a $40 \%$ nos primatas

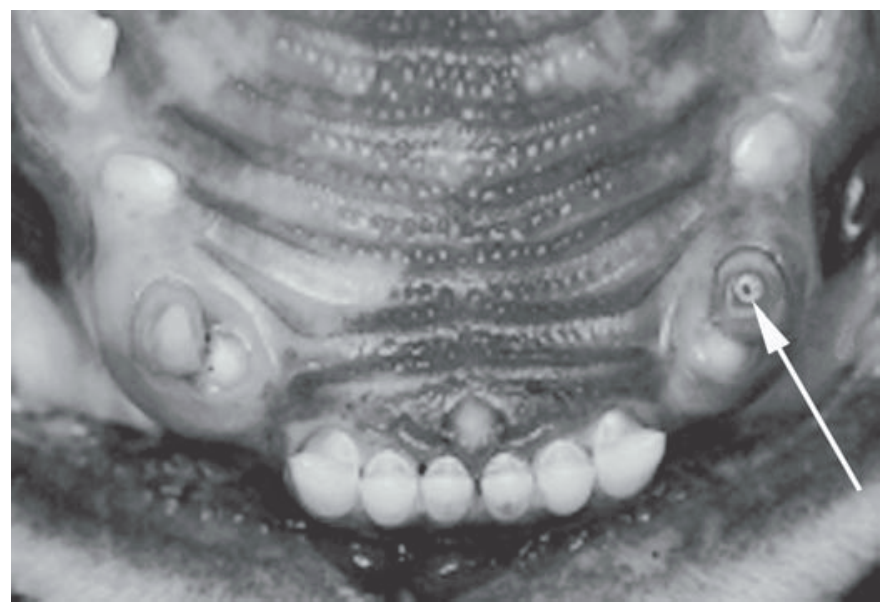

Fig.4. Início da extrusão dos dentes caninos permanentes superiores. Note-se que o dente canino superior esquerdo decíduo apresentou fratura de cúspide com exposição de canal pulpar e escurecimento dental (seta). 


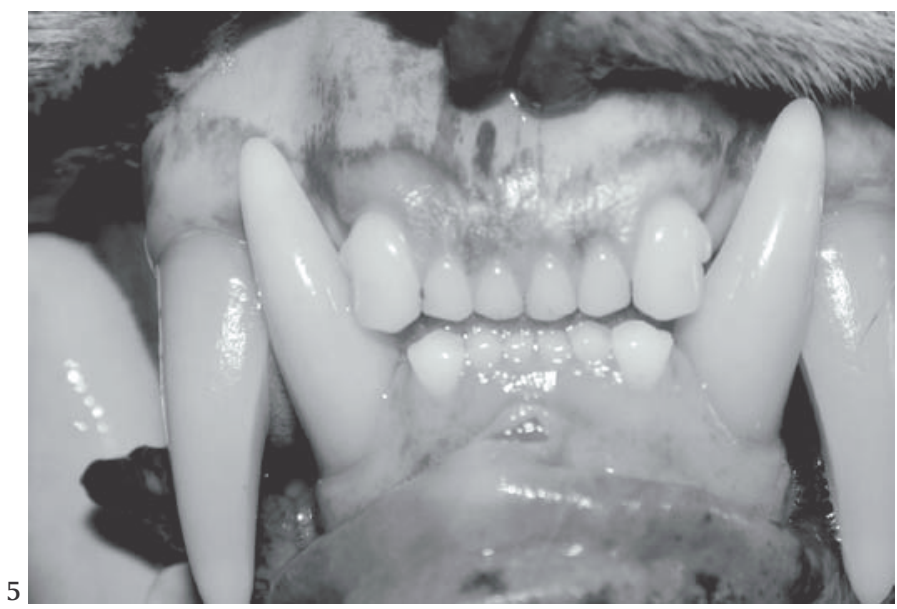

Fig.5. Oclusão rostral normal encontrada em Puma concolor (o mesmo indivíduo da Fig.3), 2 anos e 8 meses após a foto anterior.

Fig.6. Oclusão lateral esquerda normal encontrada em Puma concolor (o mesmo indivíduo da Fig.3), 2 anos e 8 meses após primeiro exame físico.

Fig.7. Oclusão lateral direita normal encontrada em Puma concolor, 2 anos e 8 meses após primeiro exame físico.

(Amand \& Tinkelman 1985). Em cativeiro, estes percentuais podem duplicar ou quadruplicar, segundo os autores.

Faltam estudos longitudinais para determinar a idade com que ocorre a esfoliação dos dentes decíduos em felídeos silvestres. Nestes, não se pode afirmar se as maloclusões foram decorrentes de acasalamentos consangüíneos (Wiggs \& Lobprise 1997), em vista de não ter sido estudada a genealogia destes indivíduos. Estudos mais aprofundados de levantamentos da prevalência de maloclusão em carnívoros selvagens necessitam ser realizados.

Tomando-se como parâmetro a idade de esfoliação e troca dos dentes decíduos dos felinos domésticos ou dos linces da América do Norte, os espécimes silvestres mantidos em cativeiro avaliados apresentaram um atraso em 2 meses neste processo, o que acarretou na erupção dos dentes caninos permanentes ocupando espaço adjacente e a maloclusão.

Os animais que apresentaram maloclusões quando filhotes aparentemente não tinham problemas de apreensão de alimentos ou de mastigação, embora apresentassem acúmulo de alimentos ou indutos moles na região dos dentes com espaço interproximal mais reduzido, o que poderia levar a problemas futuros devido à falta de remoção destes restos alimentares (doença periodontal).

\section{CONCLUSÕES}

- O Puma concolor mantido em cativeiro pode apresentar retenção de dentes decíduos;

- Grandes felinos neotropicais (Puma concolor) possuem problemas de maloclusão;

- Estudos de genealogia dos animais pode ajudar a elucidar se a maloclusão está realmente associada à consangüinidade.

- Os animais que apresentaram maloclusão não necessitaram de tratamento ortodôntico uma vez que os dentes com-
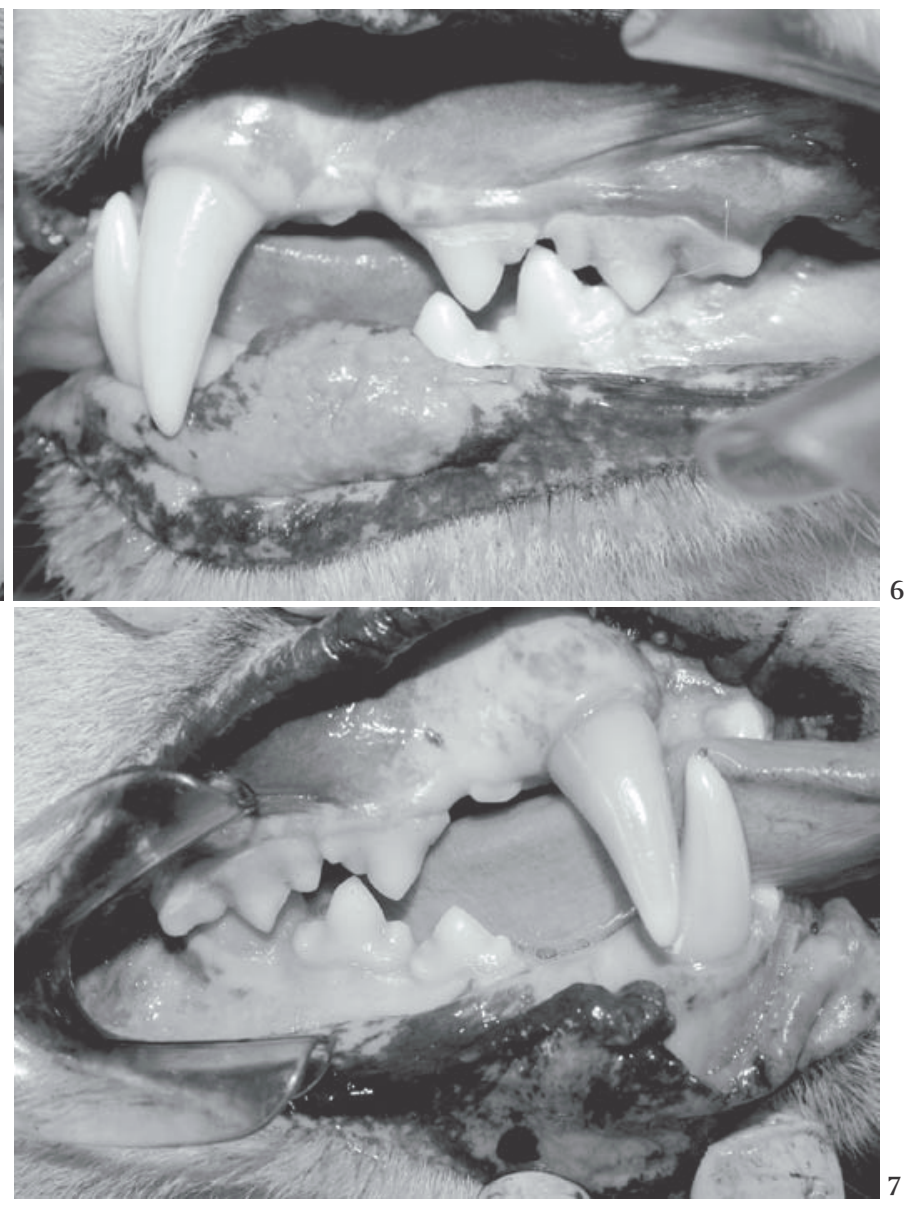

prometidos não tinham sua função mastigatória e predatória prejudicada; e

- Os dentes decíduos esfoliaram-se espontaneamente e a oclusão tornou-se normal, sem interferência profissional.

Agradecimentos.- À FAPESP pelo auxílio pesquisa 99/06173-5 e aos tratadores do zoológico municipal de Paulínia, SP.

\section{REFERÊNCIAS}

Amand W.B. \& Tinkelman C.L. 1985. Oral disease in captive wild animals, p.289308. In: Harvey C.E. (ed.), Veterinary Dentistry. Mosby-Year Book, St Louis.

Gioso M.A. 2007. Odontologia para o Clínico de Pequenos Animais. Faculdade de Medicina Veterinária e Zootecnia, Universidade de São Paulo. Editora Manole, São Paulo. 81p.

Kazimiroff T. 1938. A report on the dental pathology found in animals that diet in the New York Zoological Park in 1938. Zoologica NY Zool. Soc. 24(14):297-304.

Leite F.L.G. \& Paula T.A.R. 2002. Análise morfológica do testículo e do processo espermatogênico da onça-parda (Puma concolor, Wonzencraft 1993) adulta. Dissertação de Mestrado no Setor de Morfologia, Departamento de Medicina Veterinária, Universidade Federal de Viçosa, Minas Gerais.

Redford K.H. \& Eisenberg J.F. 1992. Mammals of the Neotropics. 2. The southern cone. University of Chicago Press, Chicago.

Robinson P.T. 1979. Oral pathology in mammals at the San Diego Zoo and Wild Animal Park. Proc. Am. Assoc. Zoo Vet. p.96-98.

Wiggs R.B. \& Lobprise H.B. 1997. Exotic animal oral disease and dentistry, p.538-56. In: Wiggs R.B. \& Lobprise H.B. (ed.), Veterinary Dentistry: principles and practice. Lippincott- Raven, New York. 